\title{
First-principles calculations of opto-electronic properties of IIIAs (III = Al, Ga, In) under influence of spin-orbit interaction effects
}

\author{
MALAK AZMAT ALI*®, NADEEM KHAN, FAROOQ AHMAD, ASGHAR ALI \\ and MUHAMMAD AYAZ \\ Department of Physics, Government Post Graduate Jahanzeb College Saidu Sharif Swat, KPK 19130, Pakistan \\ *Author for correspondence (azmatupesh@gmail.com)
}

MS received 16 April 2018; accepted 24 May 2018; published online 2 February 2019

\begin{abstract}
In this article, we present first-principles calculations for structural and opto-electric properties of IIIAs $(\mathrm{III}=\mathrm{Al}, \mathrm{Ga}, \mathrm{In})$ in the zinc-blende phase. Our calculations are based on the full potential-linearized augmented plane wave method implemented in the WIEN2k code. We employed Perdew-Burke-Ernzerhof generalized gradient and modified Becke-Johnson approximations as exchange-correlation potentials. Our calculated structure parameters are found to be in reasonable agreement with the available literature. It was found that the inclusion of spin-orbit interaction effect shifts the conduction band minima towards the Fermi level and provides band gaps very close to their experimental values. The optical properties such as complex dielectric functions, complex refractive indices, reflectivities, energy loss functions, optical conductivities and absorption coefficients at varied frequencies were investigated in detail. We found that static real part of dielectric functions and refractive index decreases with increase in band gap. Our calculated critical point energies $(\mathrm{eV})$ are consistent with the experimental results.
\end{abstract}

Keywords. FP-LAPW; structural properties; electronic properties; spin-orbit interaction; optical properties.

\section{Introduction}

Group III-V semiconductors have significant importance due to their applications in optoelectronic operating systems from ultraviolet to the infrared range [1]. These compounds can produce novel materials having adjustable electronic as well as magnetic properties [2]. They crystallize in the zinc blende (ZB) and wurtzite phases [3]. But most of group III-V semiconductors exist in the ZB structure [4], within which the band gaps for III-arsenide (IIIAs) always remain less than about $2.5 \mathrm{eV}$, and we can consider them as narrow band gap materials [5]. Due to this special property, IIIAs compounds have been received extensive interest for their significant role in the semiconductor industry. These are specially valued for their applications in optoelectronic systems and devices such as solar cells, lasers and light-emitting diodes. Moreover, AlAs and GaAs owing to their specific characteristics are used in the formation of epitaxial multilayer structures such as hetero-junction bipolar transistors, high-electron mobility transistors and Bragg reflector superlattices including solid-state lasers [6]. GaAs, a direct band gap semiconductor material, has shown its promising and efficient role in photovoltaic systems. Overall, IIIAs have a wide range of applications including the fabrication of photo-detectors, photodiodes, infraredspectrum lasers, electro-optic modulators and frequency mixing components [7].
The electronic properties of IIIAs have been studied in many experimental [8-13] and theoretical works [1,1422]. Theoretically, different approaches have been used for calculations. These approaches include local density approximation (LDA) [1], Engel-Vosko generalized-gradient approximation (EV-GGA) [14], Tran Blaha modified BeckeJohnson (TB-MBJ) approximation [14-17], Perdew-BurkeErnzerhof (PBE) approximation [17], local spin density approximation (LSDA) [18], Becke-3-Lee Yang Parr (B3LYP) approximation [18,23], Heyd-Scuseria-Ernzerhof (HSE) approximation [18,22], generalized density functional theory (GDFT) [19], Hartree-Fock approximation [20], MBJ +LDA [22] and Gowo approximation [1,21-23]. Most of these calculations are either based on semi-empirical techniques or simplified calculations that neglect some corrections like spin-orbit interaction (SOI) and orbit potential. The lack of these corrections makes the calculations easier and minimizes computational time, but the disadvantage is that the results do not match with the experimental results. IIIAs (III $=\mathrm{Al}, \mathrm{Ga}, \mathrm{In}$ ) compounds are not yet studied in the ZB phase using the full potential-linearized augmented plane wave (FP-LAPW) method with SOI. In order to explain the experimental results theoretically we studied the opto-electrical properties of these compounds with SOI. The addition of SOI provided all results close to experimental values as compared with other theoretical results. 


\section{Calculation method}

The first-principles calculations of IIIAs (III $=\mathrm{Al}, \mathrm{Ga}$, In) were performed using the FP-LAPW method implemented in the WIEN2k code [24]. In this approach, the crystal unit cell is divided into two portions. The first portion consists of non-overlapping atomic spheres which are known as Muffin Tin (MT) spheres and the second portion is interstitial region. In the two regions, the wave functions, potentials and charge density are treated differently. For our calculations, GGA of PBE [25] and MBJ potential of Trans and Blaha [26] were employed as exchange-correlation potentials. For calculation of opto-electronic properties the effect of SOI was added which uses second variational strategy to improve the results [27]. To avoid the leakage of charge from the core, the MT radii for $\mathrm{Ga}, \mathrm{In}, \mathrm{Al}$ and As were taken as 2.3, 2.48, 2.32 and 2.31 Bohr, respectively. To expand the charge density as well as the potential, the maximum angular moment $l_{\max }=10$ was used inside the atomic spheres. To obtain the suitable total energy of convergence in interstitial region, a cut-off value of $k_{\max }=7.0 / R_{\mathrm{MT}}$ was used. The charge density was restricted to $12(\mathrm{Ryd})^{1 / 2}$. A mesh of 1000 and $400 \mathrm{k}$ points in the first Brillouin zone was utilized for computation of structural and electronic properties, respectively.

\section{Results and discussion}

\subsection{Structural properties}

The ZB structure of IIIAs (III = Al, Ga, In) has a face-centred cubic lattice in which the position of group III element is $(0,0$, 0 ) and position of As is $(0.25,0.25,0.25)$ as shown in figure 1 . In our study the ground-state properties of these compounds were calculated by an optimization process in which volume is varied and energy of each volume was calculated. The ground-state volume is that one which has minimum energy. Optimized volume of unit cell was obtained by plotting the unit cell volume against total energy and using the Birch Murnaghan's equation of state [28,29]:

$$
\begin{aligned}
E(V)= & E_{0}+9 \frac{V_{0} B_{0}}{16}\left[\left\{\left(\frac{V_{0}}{V}\right)-1\right\} B_{0}^{\prime}\right. \\
& \left.+\left\{\left(\frac{V_{0}}{V}\right)^{2 / 3}-1\right\}^{2}\left\{6-4\left(\frac{V_{0}}{V}\right)\right\}^{2 / 3}\right] .
\end{aligned}
$$

The optimized plots are given in figure 2 . The ground-state lattice parameter $a$, bulk modulus $B$ and bulk modulus pressure derivative $B^{\prime}$ were calculated from optimized volume. The ground-state parameters along with other calculated experimental and theoretical values are listed in table 1. Our calculated values are in good agreement with experimental values. Furthermore, in the periodic table down group III, it is observed that the lattice constant increases due to increase in atomic number while the bulk modulus decreases.

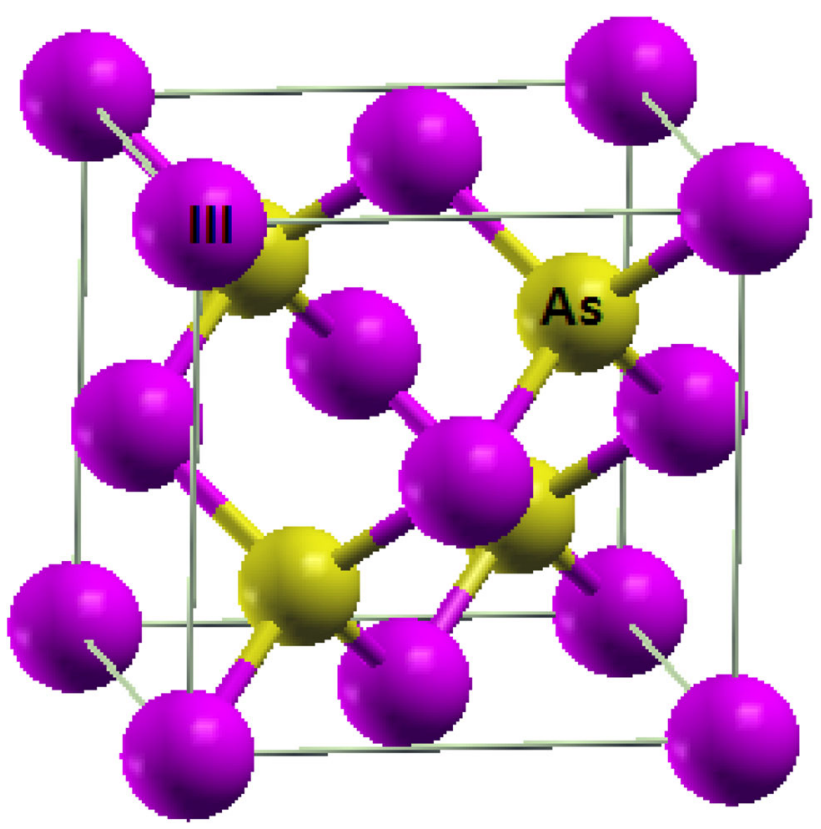

Figure 1. ZB structure of IIIAs.

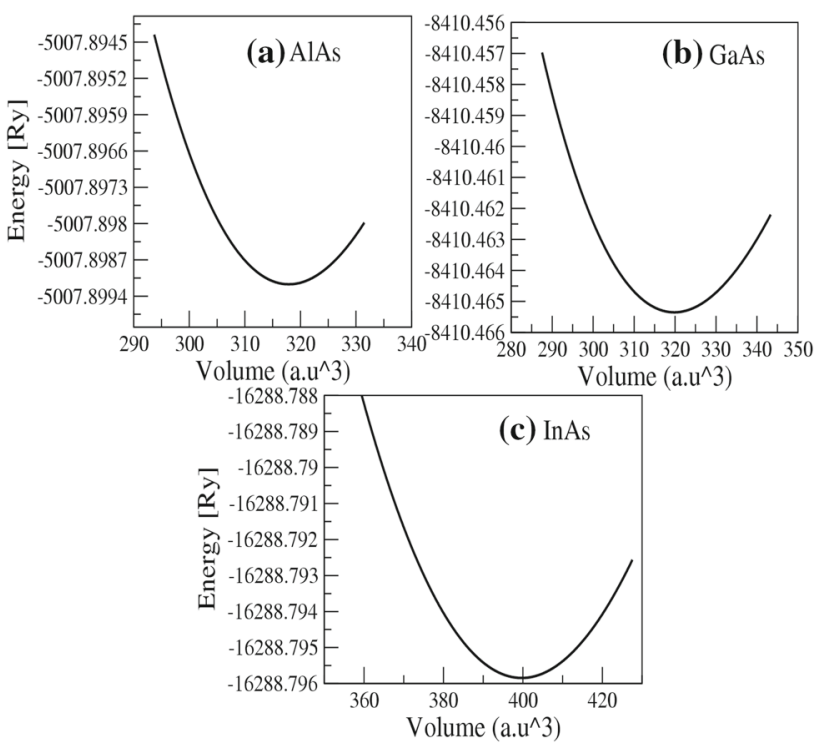

Figure 2. Calculated energy vs. volume plots for AlAs, GaAs and InAs.

\subsection{Electronic properties}

The electronic density of states (DOS) and band structures were calculated with and without inclusion of SOI effect using MBJ potential approximation and are shown in figures 3-8. DOS gives information about the contribution of electronic states in different energy levels and rough idea of the band gap of a material. Figures 3-5 show the calculated total DOSs (TDOS) of IIIAs (III = Al, Ga, In) compounds without and with the addition of SOI and partial DOSs (PDOS) with SOI. 
Table 1. Calculated ground-state lattice parameters $a(\AA)$, bulk modulus $B(\mathrm{GPa})$, bulk modulus and pressure derivative $B^{\prime}(\mathrm{GPa})$ along with experimental and other simulated results.

\begin{tabular}{|c|c|c|c|c|}
\hline Compounds & & Present & Experimental & Others \\
\hline \multirow[t]{4}{*}{ AlAs } & $a$ & 5.63 & $5.620^{\mathrm{b}}, 5.661^{\mathrm{c}}$ & $\begin{array}{l}5.614^{\mathrm{d}}(\text { PP-LDA }), 5.690^{\mathrm{m}}(\mathrm{PP}-\mathrm{GGA}), 5.664^{\mathrm{h}} \\
\quad\left(\text { FP-LDA), 5.644P }\left(\text { FP-LDA), 5.741 }{ }^{\mathrm{f}}(\mathrm{HF}), 5.76^{\mathrm{q}}\right.\right. \\
(\mathrm{HF})\end{array}$ \\
\hline & $V$ & 301.76 & & \\
\hline & $B$ & 75.56 & $82.0^{\mathrm{e}}, 77.3^{\mathrm{a}}$ & $\begin{array}{l}74.7^{\mathrm{d}}(\mathrm{PP}-\mathrm{LDA}), 71.0^{\mathrm{m}}(\mathrm{PP}-\mathrm{GGA}), 71.0^{\mathrm{h}}(\mathrm{FP}-\mathrm{LDA}) \\
75.4^{\mathrm{p}} \text { (PF-LDA), } 90.0^{\mathrm{f}}(\mathrm{HF}), 79.0^{\mathrm{q}}(\mathrm{HF})\end{array}$ \\
\hline & $B^{\prime}$ & 4.13 & $4.182^{\mathrm{e}}$ & $4.182^{\mathrm{d}}(\mathrm{PP}-\mathrm{LDA}), 4.4^{\mathrm{p}}(\mathrm{HF})$ \\
\hline \multirow[t]{4}{*}{ GaAs } & $a$ & 5.655 & $5.654^{\mathrm{a}}, 5.653^{\mathrm{k}}$ & $\begin{array}{l}5.651^{\mathrm{h}}(\text { FP-LDA }), 5.530^{\mathrm{d}}(\text { PP-LDA }), 5.700^{\mathrm{m}} \\
(\text { PP-GGA }), 5.508^{\mathrm{i}}(\text { FP-LDA }), 5.726^{\mathrm{j}}(\text { FP-GGA }) \\
5.755^{\mathrm{f}}(\mathrm{HF})\end{array}$ \\
\hline & $V$ & 305.19 & & \\
\hline & $B$ & 68.1766 & $77.0^{\mathrm{e}}, 76.0^{\mathrm{k}}$ & $\begin{array}{c}75.7^{\mathrm{d}}(\text { PP-LDA }), 65.0^{\mathrm{m}}(\text { PP-GGA }), 63.0^{\mathrm{h}}(\text { FP-LDA }), \\
77.1^{\mathrm{i}}(\text { FP-LDA }), 87.0^{\mathrm{f}}(\mathrm{HF}), 68.0^{\mathrm{j}}(\text { FP-GGA })\end{array}$ \\
\hline & $B^{\prime}$ & 4.47 & $4.487^{\mathrm{e}}$ & $4.487^{\mathrm{d}}(\mathrm{PP}-\mathrm{LDA}), 4.8^{\mathrm{p}}$ (FP-LDA), $4.46^{\mathrm{j}}$ (FP-LDA) \\
\hline \multirow[t]{4}{*}{ InAs } & $a$ & 6.1 & $6.036^{\mathrm{b}}$ & $\begin{array}{l}5.921^{\mathrm{d}}(\mathrm{PP}-\mathrm{LDA}), 5.902^{\mathrm{g}}(\mathrm{PP}-\mathrm{LDA}), 6.015^{\mathrm{p}} \\
\quad(\mathrm{FP}-\mathrm{LDA}), 6.152^{\mathrm{f}}(\mathrm{HF}), 6.15^{\mathrm{q}}(\mathrm{HF})\end{array}$ \\
\hline & $V$ & 398.73 & & \\
\hline & $B$ & 52.58 & $58.0^{\mathrm{e}}$ & $\begin{array}{l}61.7^{\mathrm{d}}(\mathrm{PP}-\mathrm{LDA}), 61.9^{\mathrm{g}}(\mathrm{PP}-\mathrm{LDA}), 60.3^{\mathrm{p}}(\text { FP-LDA }), \\
74.0^{\mathrm{f}}(\mathrm{HF}), 64.0^{\mathrm{q}}(\mathrm{HF})\end{array}$ \\
\hline & $B^{\prime}$ & 4.75 & $4.79^{\mathrm{n}}$ & $4.545^{\mathrm{d}}$ (PP-LDA), $4.488^{\mathrm{g}}$ (PP-LDA), $4.9^{\mathrm{p}}$ (FP-LDA) \\
\hline
\end{tabular}

${ }^{a}$ Ref.[3], ${ }^{b}$ ref.[30], ${ }^{c}$ ref.[9], ${ }^{d}$ ref.[31], ${ }^{\mathrm{e}}$ ref. [32], ${ }^{\mathrm{f}}$ ref. [33], ${ }^{\mathrm{g}}$ ref. [34], ${ }^{\mathrm{h}}$ ref. [35], ${ }^{\mathrm{i}}$ ref. [36], ${ }^{\mathrm{j}}$ ref. [37], ${ }^{\mathrm{k}}$ ref. [38], ${ }^{\mathrm{r}}$ ref. [39], ${ }^{\mathrm{m}}$ ref. [40], ${ }^{\mathrm{n}}$ ref. [41], ${ }^{\mathrm{o}}$ ref. [42], pref. [43], ${ }^{\mathrm{q}}$ ref. [44].

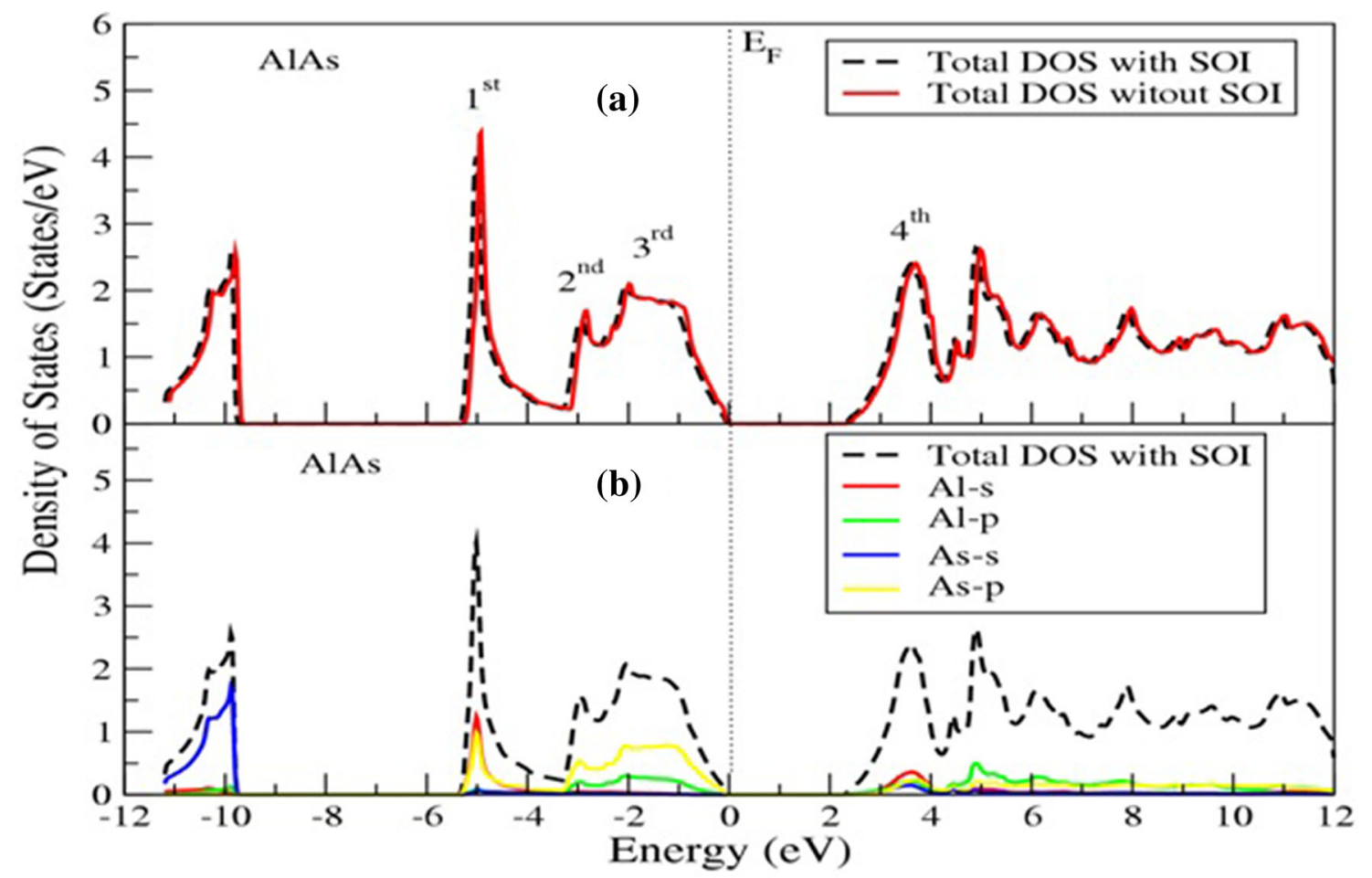

Figure 3. DOS of AlAs: (a) comparison between the total DOS with and without SOI and (b) total and partial DOS with SOI. 
Panel (a) of figures shows comparison between the TDOS with and without SOI, from which it can be deduced that DOS can be divided into four major peaks. The first three peaks contribute to the valence band (VB) and the fourth peak lies to the right side of the Fermi level and contributes to the conduction band (CB) maximum. When SOI is applied, a shift in the fourth peak is observed towards the Fermi level which reduces the forbidden energy gap. The electronic transitions actually occur between the maximum of VB and the minimum of $\mathrm{CB}$, therefore, shift of the fourth peak is more significant as compared with other peaks. The reason of shifting can be explained as follows. In the electron's frame of reference the nucleus can be treated as making a current loop

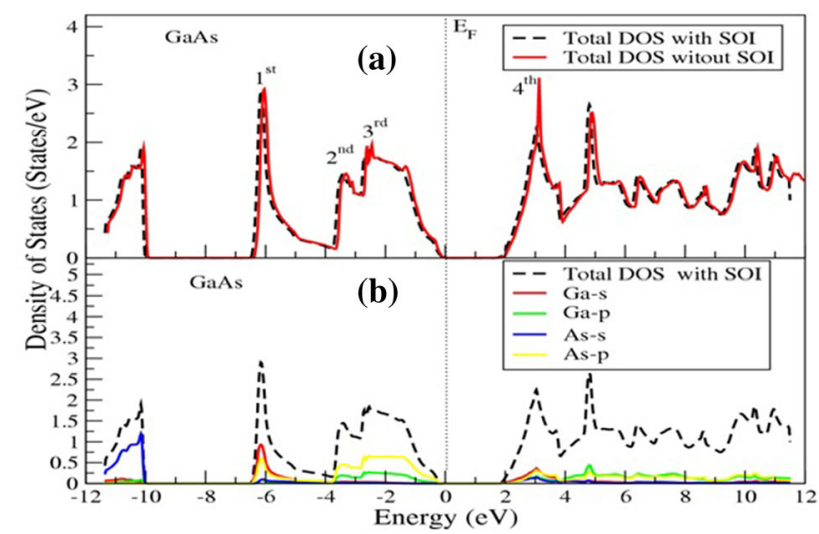

Figure 4. DOS of GaAs: (a) comparison between the total DOS with and without SOI and (b) total and partial DOS with SOI. around the electron. Thus electrons exist in the magnetic field of the current loop. The spin magnetic moment interacts with the magnetic field established by the orbital motion of the nucleus which results in the removal of degeneracy and splits the energy states [27]. The resulting states repel each other and reduces band gap.

Panel (b) in figures 3-5 shows the contribution of sub-energy levels to TDOS of AlAs, GaAs and InAs, respectively. Panel (b) of all figures shows that VB minimum has major contribution of $s$ state of As and have minor contribution of $s$ and $p$ states of group III elements along with $p$ state of As. VB maximum is majorly composed of $p$ states of As along with minor contribution of $s$ and $p$ states of group III elements and s state of As. In the CB maximum the s state of group III elements has greater contribution and $p$ state of group III elements along with $\mathrm{s}$ and $\mathrm{p}$ state of As has minor contribution while the CB minimum is mainly made of $p$ state of group III elements along with minor contribution of s state of group III elements and $\mathrm{s}$ and $\mathrm{p}$ states of As.

The DOS plots give us the general idea of the band gap. However, to know the exact nature of band gap, we have to find variations of different energy levels along with symmetry points in the first Brillion zone. The calculated electronic band structures without and with SOI are shown in figures 6-8. The VB maximum and CB minimum lies at the same symmetry point $\Gamma$ for GaAs and InAs, while the VB maxima for AlAs lies at $\Gamma$ point and $C B$ minima lies at $X$ point. Hence, the fundamental band gap of GaAs and InAs is direct and AlAs is indirect. Our simulated band gaps along with other theoretical and experimental results are given in table 2. Our calculated

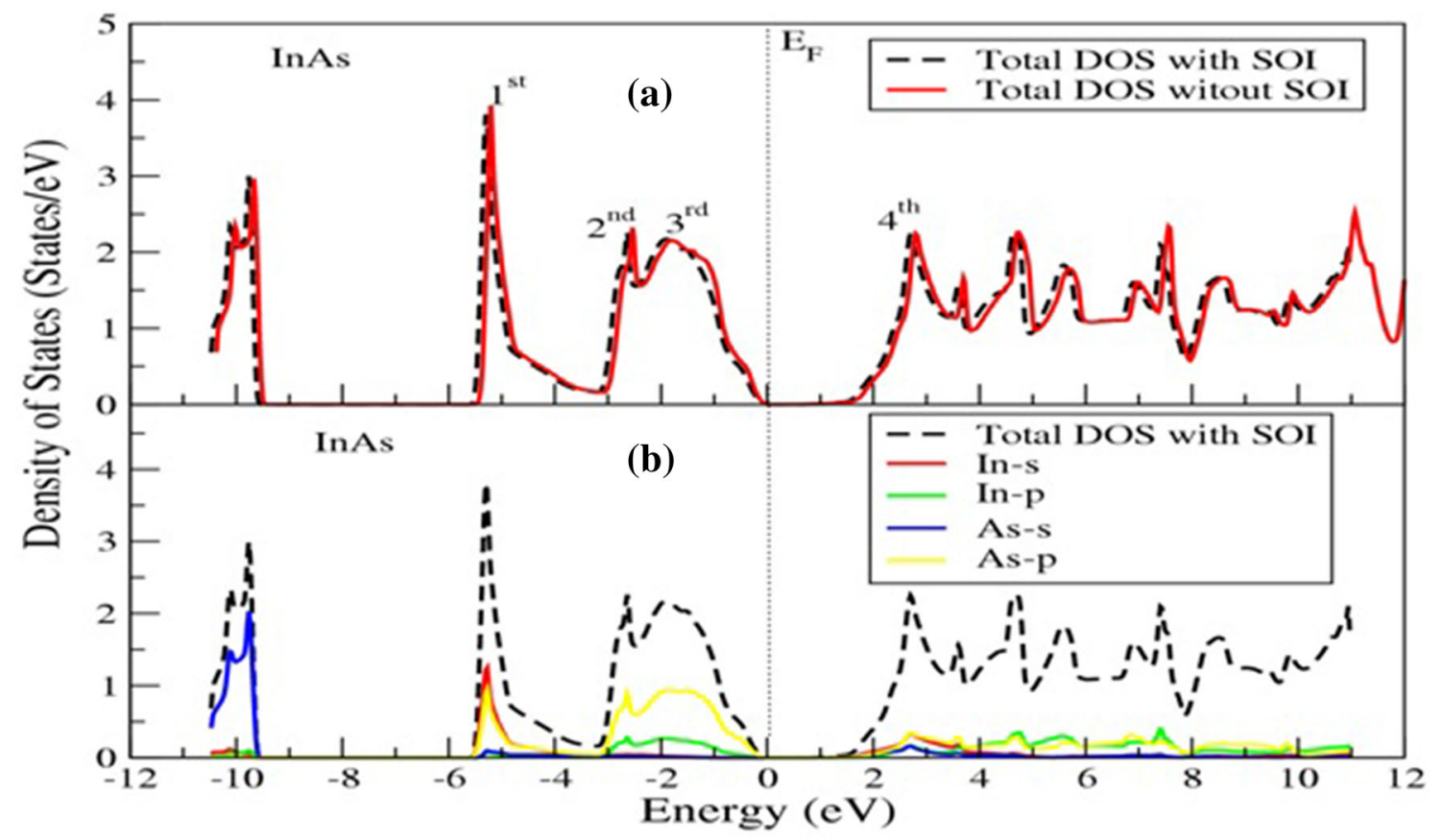

Figure 5. DOS of InAs: (a) comparison between the total DOS with and without SOI and (b) total and partial DOS with SOI. 

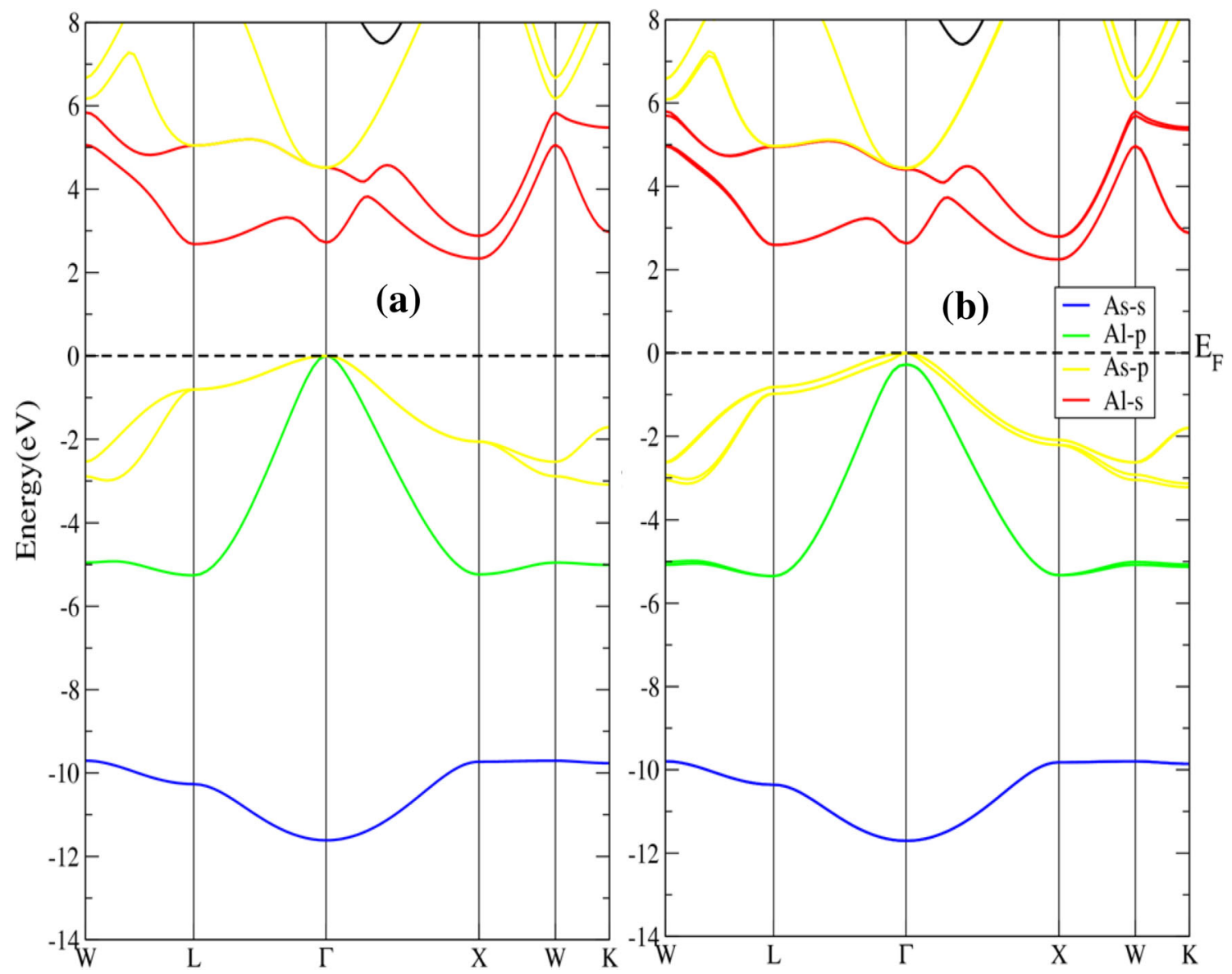

Figure 6. Band gap of AlAs: (a) without SOI and (b) with SOI.

results are much closer to experimental results as compared with other previously published simulated results. The addition of SOI causes the splitting of As-p states at symmetry points $\mathrm{W}, \mathrm{L}, \mathrm{X}$ and $\Gamma$ in the upper VB and the splitting of Al-s, Ga-s and In-s states at symmetry points $\mathrm{L}, \Gamma$ and $\mathrm{W}$ in the lower CB for AlAs, GaAs and InAs, respectively. This splitting causes repulsion between energy states which results in the shifting of Al-s states along the X point, and Ga-s and In-s states along the $\Gamma$ point towards lower energy states. Due to splitting and shifting, reduced band gaps of all studied compounds were obtained, which lie within the experimental range.

\subsection{Optical properties}

The optical properties of IIIAs (III $=\mathrm{Al}, \mathrm{Ga}$, In) were calculated using MBJ approximation with effect of SOI. The results were obtained without applying any broadening or scissor operators often employed to match theoretical calculations with experimental results [27]. The calculated optical spectral critical points along with other theoretical and experimental results are shown in table 3. Our calculated values are in excellent agreement with experimental results and are better than other calculated results.

3.3a Dielectric function: The dielectric function $\varepsilon(\omega)$ describes the optical response to all photon energies and is given by [48]

$$
\varepsilon(\omega)=\varepsilon_{1}(\omega)+i \varepsilon_{2}(\omega) .
$$

The real part $\varepsilon_{1}(\omega)$ shows dispersive behaviour and imaginary part $\varepsilon_{2}(\omega)$ shows absorptive behaviour of the material. The real and imaginary parts of dielectric function up to $12 \mathrm{eV}$ energy are plotted in figure 9a. The calculated zero-frequency limit, $\varepsilon_{1}(0)$ of AlAs, GaAs and InAs are 6.16, 9.14 and 9.54, respectively, which show that $\varepsilon_{1}(0)$ increases, when III goes down in the group of the periodic table from $\mathrm{Al}$ to In. Keeping this special behaviour and table 2 in mind, we can conclude that $\varepsilon_{1}(0)$ varies inversely with band gap for these materials. A similar behaviour was also discussed in other materials [49,50]. Beyond zero frequency limits, $\varepsilon_{1}(\omega)$ increases to maximum value 13.21 for AlAs, 15.02 for GaAs and 15.13 for InAs at their respective peak $E_{1}$. After maximum peaks, 

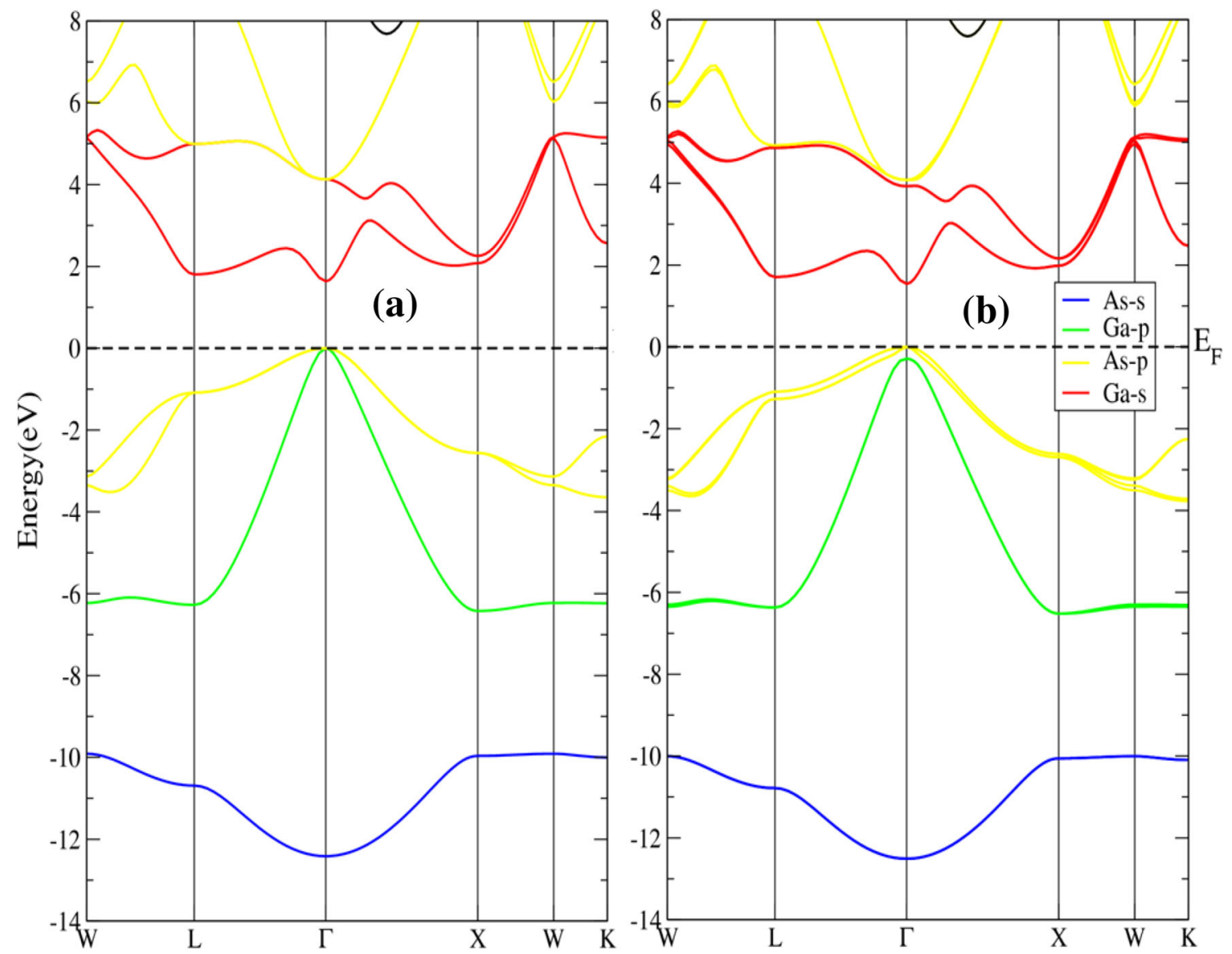

Figure 7. Band gap of GaAs: (a) without SOI and (b) with SOI.

$\varepsilon_{1}(\omega)$ decreases sharply below zero to the minimum value of -2.7 for AlAs, -4.9 for GaAs and -5.1 for InAs. For these negative values of $\varepsilon_{1}(\omega)$, the compounds lose dielectric property and show metallic character [51,52]. $\varepsilon_{2}(\omega)$ is related to the band gap of a material. Hence, for all three compounds, $\varepsilon_{2}(\omega)$ starts at our calculated band gap $\mathrm{E}_{0}$ and increase with increase in energy. The maximum value of $\varepsilon_{2}(\omega)$ occurs at peak $E_{2}$ and then reaches zero by passing through another peak $\mathrm{E}_{3}$. For AlAs, GaAs and InAs, the calculated maximum values of $\varepsilon_{2}(\omega)$ are 18.37, 15.89 and 13.86, respectively.

3.3b Complex refractive index: The complex refractive index $\tilde{n}(\omega)$ is related to refraction and absorption of light by a material and is given by [48]

$$
\tilde{n}(\omega)=n(\omega)+i K(\omega) .
$$

The real part $n(\omega)$ is called the refractive index shown by the solid line and imaginary part $K(\omega)$ is called the extinction coefficient shown by the dashed line in figure $9 \mathrm{~b}$. The value of the refractive index starts at zero frequency having a value of 2.46 for AlAs, 2.80 for GaAs and 3.07 for InAs.
The refractive index shows a similar behaviour to $\varepsilon_{1}(\omega)$, the value of $n(0)$ decreases with increase of band gap of these compounds. Beyond $n(0)$ limit, the value of $n(\omega)$ increases and reaches the maximum value of 3.93 for AlAs, 3.965 for GaAs and 4.00 for InAs, at their first peak $E_{1}$. After maximum value, $n(\omega)$ decreases rapidly near $\mathrm{E}_{2}$ and approaches to minimum values for higher energies. The extinction coefficient $K(\omega)$ shows similar behaviour to $\varepsilon_{2}(\omega)$.

3.3c Optical conductivity: When light of specific frequency falls on a material, conduction of electrons occurs. This property of a material is called optical conductivity $\sigma(\omega)$ and is given by [48]

$$
\sigma(\omega)=\frac{2 W_{\mathrm{cv}} \hbar \omega}{\vec{E}_{0}^{2}}
$$

where $W_{\text {cv }}$ is the transition probability per unit time.

The simulated plots of optical conductivity $v s$. incident photon energy with the effect of SOI are given in figure 10a. The optical conductivity $\sigma(\omega)$ like absorption coefficient for AlAs, GaAs and InAs starts at fundamental band gaps and 

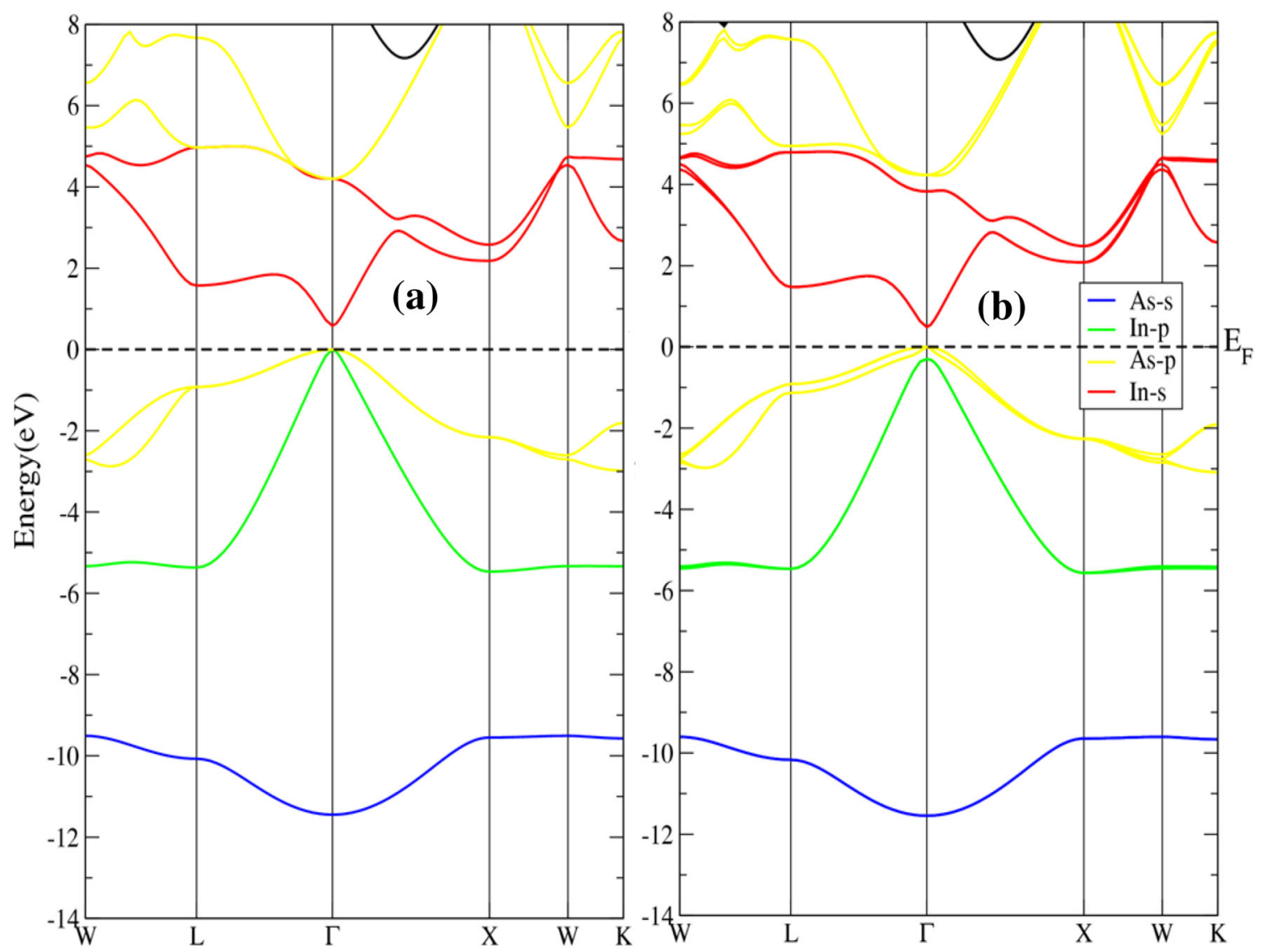

Figure 8. Band gap of InAs: (a) without SOI and (b) with SOI.

then increases at $E_{1}$ then sharply increases at $E_{2}$ and then decreases at $\mathrm{E}_{3}$. The maximum optical conductivity ranges for investigated compounds IIIAs (III = Al, Ga, In) are $4.65-5.602,4.48-5.06$ and 4.05-4.86, respectively. In the maximum conductivity ranges these materials can be best utilized in solar cells and detectors in the ultra-violet energy range. $\sigma(\omega)$ has maximum value of $1.1795 \times 10^{4} \Omega^{-1} \mathrm{~cm}^{-1}$ for AlAs at $E_{2}, 1.0007 \times 10^{4} \Omega^{-1} \mathrm{~cm}^{-1}$ for GaAs at $E_{2}$ and $8.385 \times 10^{3} \Omega^{-1} \mathrm{~cm}^{-1}$ for InAs at the second peak.

3.3d Absorption coefficient: The absorption coefficient is the property of material to absorb photons of specific energy and is given by [48]

$$
\alpha(\omega)=\frac{4 \pi K(\omega)}{\lambda}
$$

The absorption coefficient calculated with SOI is shown in figure 10b. The figure shows that absorption for all of our studied compounds starts at their fundamental band gaps. While at peak $E_{1}$ the transition from As-p states in the VB to Al-s, Ga-s and In-s states in the CB of AlAs, GaAs and InAs, respectively, occurs which is designated as $\mathrm{L}_{6,7}^{\mathrm{V}} \rightarrow \mathrm{L}_{7}^{\mathrm{C}}$. The second peak $\mathrm{E}_{2}$ for AlAs, GaAs and InAs corresponds to maximum absorption $144.6 \times 10^{4} \mathrm{~cm}^{-1}, 165.4 \times 10^{4} \mathrm{~cm}^{-1}$ and $162.3 \times 10^{4} \mathrm{~cm}^{-1}$, respectively, which shows transition $\mathrm{X}_{6,7}^{\mathrm{V}} \rightarrow \mathrm{X}_{7}^{\mathrm{C}}$ and the third peak $\mathrm{E}_{3}$ for all studied compounds corresponds to transition $\mathrm{W}_{6,7}^{\mathrm{V}} \rightarrow \mathrm{W}_{7}^{\mathrm{C}}$.

3.3e Reflectivity: The reflection of incident light from the surface of a material corresponds to reflectivity and is given by [48]

$$
R(\omega)=\left(\frac{|1-n(\omega)|}{|1+n(\omega)|}\right)
$$

The reflectivity $R(\omega)$ is plotted against the incident photon energy with addition of SOI in figure 10c. At zero frequency the reflectivity is called static reflectivity $R(0)$ having the value of $17.9 \%$ for AlAs, $22.6 \%$ for GaAs and $26.1 \%$ for InAs. In figure 10c the different peaks show variation in reflectivity when the energy increases. The maximum value of reflectivity for AlAs is $53 \%$ at $11.058 \mathrm{eV}, \mathrm{GaAs}$ is $61.2 \%$ at $11.93 \mathrm{eV}$ and for InAs is $60.7 \%$ at $10.582 \mathrm{eV}$. It is observed that the maximum value of reflectivity occurs at point where $\varepsilon_{1}(\omega)$ becomes negative because in this energy range the material shows a metallic character [53]. 
Table 2. Fundamental band gaps in eV calculated without and with SOI for GaAs and InAs compared with previously calculated works and experimental values.

\begin{tabular}{|c|c|c|c|c|}
\hline \multirow[b]{2}{*}{ Compounds } & \multicolumn{2}{|c|}{ Present } & \multirow[b]{2}{*}{ Experimental } & \multirow[b]{2}{*}{ Other theoretical works } \\
\hline & Without SOI & With SOI & & \\
\hline AlAs & 2.32 & 2.23 & $\begin{array}{c}2.24^{\mathrm{a}} \\
2.32^{\mathrm{b}} \\
2.3^{\mathrm{c}}\end{array}$ & $\begin{array}{c}1.31^{\mathrm{a}}(\mathrm{LDA}), 1.40^{\mathrm{b}}(\mathrm{GGA}), \\
2.25^{\mathrm{c}}(\mathrm{EV}-\mathrm{GGA}), 1.98^{\mathrm{d}}(\mathrm{MBJ}) \\
2.12^{\mathrm{c}}(\mathrm{TB}-\mathrm{MBJ}), 2.25^{\mathrm{c}}(\mathrm{nTB}-\mathrm{MBJ}) \\
2.06^{\mathrm{d}}(\mathrm{MBJ}), 2.12^{\mathrm{d}}(\mathrm{MBJ}+\mathrm{PBE}) \\
2.71^{\mathrm{e}}(\mathrm{B} 3 \mathrm{LYP}), 2.24^{\mathrm{e}}(\mathrm{HSE}) \\
1.40^{\mathrm{e}}(\mathrm{LSDA}), 2.54^{\mathrm{g}}(\mathrm{LDA}) \\
1.58^{\mathrm{g}}(\mathrm{GW}), 2.73^{\mathrm{h}}(\mathrm{GW}) \\
2.48^{\mathrm{i}}(\mathrm{LDA}), 3.26^{\mathrm{k}}(\mathrm{GW}) \\
2.29^{\mathrm{k}}(\mathrm{LDA}), 3.3^{\mathrm{l}}(\mathrm{B} 3 \mathrm{LYP}) \\
2.24^{\mathrm{l}}(\mathrm{HSE})\end{array}$ \\
\hline GaAs & 1.61 & 1.52 & $\begin{array}{l}1.42^{\mathrm{a}} \\
1.52^{\mathrm{b}}\end{array}$ & $\begin{array}{c}0.28^{\mathrm{a}}(\mathrm{LDA}), 0.25^{\mathrm{a}}(\mathrm{GGA}) \\
1.03^{\mathrm{a}}(\mathrm{EV}-\mathrm{GGA}), 1.56^{\mathrm{b}}(\mathrm{MBJ}) \\
1.6^{\mathrm{c}}(\mathrm{TB}-\mathrm{MBJ}), 1.51^{\mathrm{c}}(\mathrm{nTB}-\mathrm{MBJ}) \\
1.55^{\mathrm{d}}(\mathrm{MBJ}), 1.44^{\mathrm{d}}(\mathrm{MBJ}+\mathrm{PBE}) \\
1.44^{\mathrm{e}}(\mathrm{B} 3 \mathrm{LY}), 1.21^{\mathrm{e}}(\mathrm{HSE}) \\
0.43^{\mathrm{e}}(\mathrm{LSDA}), 1.06^{\mathrm{f}}(\mathrm{LDA}) \\
1.58^{\mathrm{f}}(\mathrm{GW}), 1.60^{\mathrm{i}}(\mathrm{LDA}) \\
1.33^{\mathrm{j}}(\mathrm{HSE} 06), 1.56^{\mathrm{j}}(\mathrm{MBJLDA}) \\
0.43^{\mathrm{j}}(\mathrm{PBE}), 1.47^{\mathrm{k}}(\mathrm{GW}) \\
0.56^{\mathrm{k}}(\mathrm{LDA}), 1.91^{1}(\mathrm{~B} 3 \mathrm{LYP}) \\
1.21^{\mathrm{l}}(\mathrm{HSE})\end{array}$ \\
\hline InAs & 0.56 & 0.47 & $\begin{array}{l}0.42^{\mathrm{a}} \\
0.42^{\mathrm{b}}\end{array}$ & $\begin{array}{c}0.00^{\mathrm{a}}(\mathrm{LDA}), 0.00^{\mathrm{a}}(\mathrm{GGA}) \\
0.40^{\mathrm{a}}(\mathrm{EV}-\mathrm{GGA}), 0.60^{\mathrm{b}}(\mathrm{MBJ}) \\
0.66^{\mathrm{c}}(\mathrm{TB}-\mathrm{MBJ}), 0.42^{\mathrm{c}}(\mathrm{nTB}-\mathrm{MBJ}) \\
0.57^{\mathrm{d}}(\mathrm{MBJ}), 0.46^{\mathrm{d}}(\mathrm{MBJ}+\mathrm{PBE}) \\
0.55^{\mathrm{e}}(\mathrm{B} 3 \mathrm{LYP}), 0.39^{\mathrm{e}}(\mathrm{HSE}) \\
0.00^{\mathrm{e}}(\mathrm{LSDA}), 0.25^{\mathrm{g}}(\mathrm{LDA}) \\
0.31^{\mathrm{g}}(\mathrm{GW}), 0.46^{\mathrm{h}}(\mathrm{GW}) \\
0.42^{\mathrm{j}}(\mathrm{HSE} 06), 0.61^{\mathrm{j}}(\mathrm{MBJLDA}) \\
-0.30^{\mathrm{j}}(\mathrm{PBE})\end{array}$ \\
\hline
\end{tabular}

${ }^{a}$ Ref. [14], ${ }^{b}$ ref. [15], ${ }^{c}$ ref. [16], ${ }^{d}$ ref. [18], ${ }^{\mathrm{e}}$ ref. [19], ${ }^{\mathrm{f}}$ ref. [45], ${ }^{\mathrm{g}}$ ref. [19], ${ }^{\mathrm{h}}$ ref. [21], ${ }^{\mathrm{i}}$ ref. [1], ${ }^{\mathrm{j}}$ ref. [22], ${ }^{\mathrm{k}}$ ref [23], ${ }^{\mathrm{l}}$ ref [20].

From the reflectivity curves the three minima for AlAs occurs at $6.28,8.25$ and $9.48 \mathrm{eV}$, for GaAs occurs at 5.58 , 6.92 and $8.88 \mathrm{eV}$ and for InAs occurs at 5.24, 7.91 and $9.59 \mathrm{eV}$. These minima are related to transmission resonance frequencies.

3.3f Energy loss spectrum: The resonance of plasma is related to energy loss function which corresponds to the frequency of incident radiation. The energy loss function vs. incident light for AlAs, GaAs and InAs with addition of SOI is plotted in figure $10 \mathrm{~d}$. The plots show three peaks for AlAs at $6.28,8.25$ and $9.48 \mathrm{eV}$ corresponding to frequencies $1.51 \times 10^{15}, 1.99 \times 10^{15}$ and $2.28 \times 10^{15} \mathrm{~Hz}$, three peaks for GaAs at energy 5.58, 6.92 and $8.88 \mathrm{eV}$ corresponding to frequencies $1.34 \times 10^{15}, 1.66 \times 10^{15}$ and $2.14 \times 10^{15} \mathrm{~Hz}$ and three peaks for InAs at 5.24, 7.91 and $9.59 \mathrm{eV}$ corresponding to frequencies $1.26 \times 10^{15}, 1.90 \times 10^{15}$ and $2.31 \times 10^{15} \mathrm{~Hz}$, respectively. Above these frequencies the transmittance of
Table 3. Comparison of calculated and experimental values of characteristic peaks in optical spectra in $\mathrm{eV}$.

\begin{tabular}{lcccc} 
Compounds & Peaks & This work & Other works & Experimental \\
\hline AlAs & $\mathrm{E}_{1}$ & 4.3 & $3.5^{\mathrm{a}}, 4.05^{\mathrm{f}}$ & $3.8^{\mathrm{g}}, 3.90^{\mathrm{g}}$ \\
& $\mathrm{E}_{2}$ & 4.78 & $4.4^{\mathrm{a}}, 5.03^{\mathrm{f}}$ & $4.85^{\mathrm{g}}, 4.89^{\mathrm{g}}$ \\
& $\mathrm{E}_{3}$ & 5.81 & & \\
GaAs & $\mathrm{E}_{1}$ & 3.06 & $2.9^{\mathrm{a}}, 3.16^{\mathrm{b}}$ & $3.017^{\mathrm{c}}, 2.90^{\mathrm{d}}$ \\
& $\mathrm{E}_{2}$ & 4.72 & $4.4^{\mathrm{a}}, 4.83^{\mathrm{b}}$ & $5.11^{\mathrm{c}}, 4.75^{\mathrm{d}}$ \\
& $\mathrm{E}_{3}$ & 6.11 & & \\
InAs & $\mathrm{E}_{1}$ & 2.55 & $2.6^{\mathrm{a}}$ & $2.5^{\mathrm{d}}, 2.6^{\mathrm{e}}$ \\
& $\mathrm{E}_{2}$ & 4.45 & $4.3^{\mathrm{a}}$ & $4.45^{\mathrm{d}}, 4.3^{\mathrm{e}}$ \\
& $\mathrm{E}_{3}$ & 5.78 & & \\
\hline
\end{tabular}

${ }^{a}$ Ref. [8], ${ }^{b}$ ref. [46], ${ }^{c}$ ref. [12], ${ }^{d}$ ref. [9], ${ }^{\mathrm{e}}$ ref. [13], ${ }^{\mathrm{f}}$ ref. [47], ${ }^{\mathrm{g}}$ ref. [11].

materials increases and shows transparent behaviour [54] because the maxima of energy loss function corresponds to minima of reflectivity. 

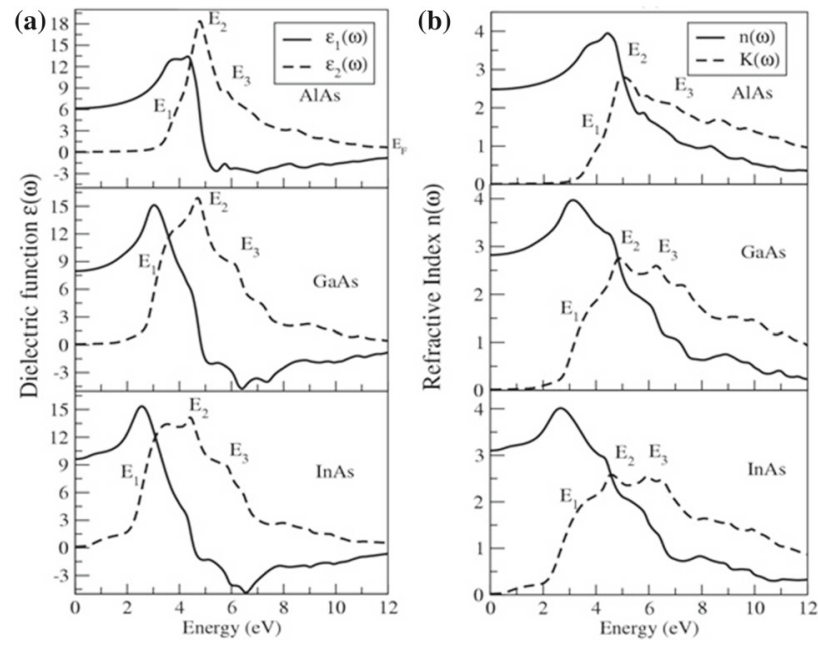

Figure 9. (a) Dielectric function and (b) refractive index for AlAs, GaAs and InAs.
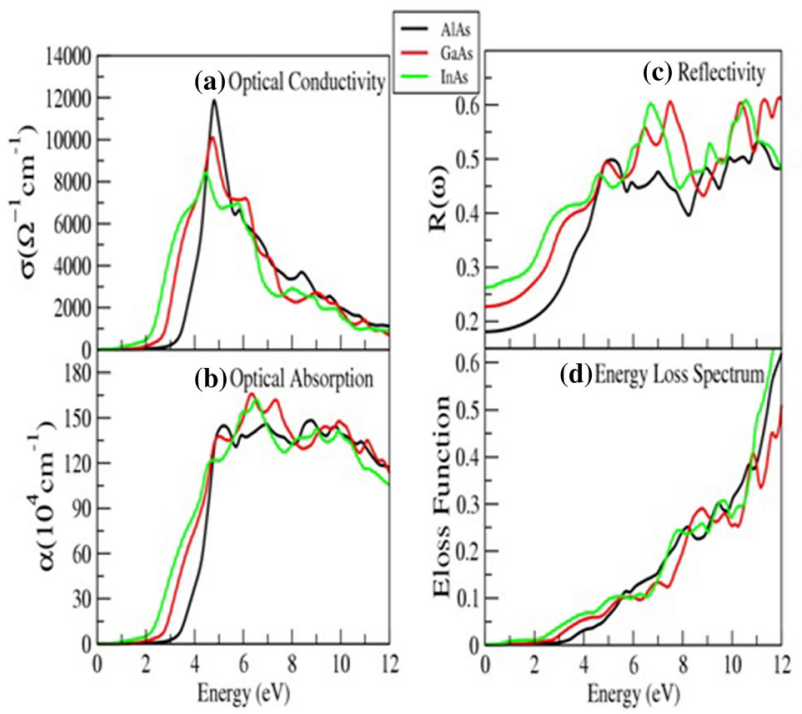

Figure 10. (a) optical conductivity, (b) optical absorption, (c) reflectivity and (d) energy loss spectrum for AlAs, GaAs and InAs.

\section{Conclusions}

In this study, we calculated the structural, electronic and optical properties of IIIAs (III $=\mathrm{Ga}$, In, $\mathrm{Al}$ ) using the WIEN2k code based on DFT. For calculation of electronic and optical properties the SOI effect was included. The calculated ground-state properties are in good agreement with experiment. It was noted that when group III element changes from $\mathrm{Al}$ to In, the lattice parameters and derivative of bulk modulus increases while bulk modulus decreases. SOI effect breaks the degeneracy and splits the electronic states. The repulsion between the resulting states shifts CB minima towards the Fermi level, due to which reduced band gaps of AlAs
$(\Gamma-\mathrm{X})$, GaAs $(\Gamma-\Gamma)$ and InAs $(\Gamma-\Gamma)$ were obtained which are comparable with experimental results. The static real part of complex dielectric function and refractive index decreases as band gap increases. Optical conductivity decreases by going from $\mathrm{Al}$ to In. Maxima of energy loss function corresponds to minima of reflectivity and in such situation IIIAs compounds show transparent behaviour. From the optical spectra analysis these materials are predicted to be useful for optical applications in the infrared, visible and ultraviolent energy ranges.

\section{References}

[1] Ribeiro M, Fonseca L R C and Ferreira L G 2011 EPL 9427001

[2] Annane F, Meradji H, Ghemid S and Hassan F E H 2010 Comput. Mater. Sci. 50274

[3] Vurgaftman I, Meyer J R and Mohan L R R 2001 Appl. Phys. Rev. 895815

[4] Rehamn G, Shafiq M, Saifullah M, Ahmad M, Asadadabadi S J, Maqbool M et al 2016 J. Electron. Mater. 453314

[5] Ziane M I, Bensaad Z, Labdelli B and Bennacer H 2014 Sens. Transducers J. 27374

[6] Ferreira L, Wei S H and Zunger A 1998 Phys. Rev. B 403197

[7] Haq B U, Ahmad R, Hassan F E H, Khenata R, Kasim M K and Goumri-Said S 2014 Sol. Energy 1001

[8] Fern R E and Onton R E 1971 J. Appl. Phys. 423499

[9] Adachi S 1987 Phys. Rev. B 357454

[10] Joyce J H, Docherty C J, Gao Q, Tan H H, Jagadish C, Hughes J L et al 2013 Nanotechnology 24214006

[11] Garriga M, Lautenschlager P, Cardona M and Ploog K 1987 Solid State Commun. 63157

[12] Yu P Y and Cardona M 2010 Fundamentals of semiconductors physics, 4th edition (Netherland: Springer)

[13] Djurisic A B, Rakic A D, Kwok P C K, Li E H and Majewski M L 1999 J. Appl. Phys. 853638

[14] Ahmed R, Hashemifar S J, Akbarzadeh H, Ahmad M and Aleem F 2007 Comput. Mater. Sci. 39580

[15] Koller D, Tran F and Blaha P 2005 Phys. Rev. B 72193201

[16] Wang Y, Yin H, Cao R, Zahid F, Zhu Y, Liu L et al 2013 Phys. Rev. B 87235203

[17] Hinuma Y, Gruneis A, Kresse G and Oba F 2014 Phys. Rev. B 90155405

[18] Tomic S, Montanri B and Harrison N M 2008 J. Physica E 40 2125

[19] Remediakis I N and Kaxiras E 1999 Phys. Rev. B 595536

[20] Shimazaki T and Asai Y 2010 J. Chem. Phys. 13016401

[21] Lebegue S, Arnaud B, Alouani M and Bloech P E 2003 Phys. Rev. B 67155208

[22] Kim Y S, Marshman M, Kresse G, Tran F and Blaha P 2010 Phys. Rev. B $\mathbf{8 2} 205212$

[23] Godby R W, Schlutter M and Sham L J 1987 Phys. Rev. B 35 4170

[24] Blaha P, Schwarz K, Madsen G K H, Kvasnicka D and Luitz J 2001 WIEN2K, An augmented plane wave plus local orbitals program for calculating crystal properties (Vienna, Austria: Vienna University of Technology)

[25] Wong K M, Alay-e-Abbas S M, Fang Y, Shaukat A and Lei Y 2013 J. Appl. Phys. 114034901 
[26] Tran F and Blaha P 2009 Phys. Rev. Lett. 102226401

[27] Hilal M, Rashid B, Khan S H and Khan A 2016 Mater. Chem. Phys. 18441

[28] Ali M A, Khan A, Khan S H, Ouohrani T, Murtaza G, Khenata R et al 2015 Mater. Sci. Semicond. Process. 3857

[29] Faizan M, Murtaza G, Khan S H, Khan A, Mehmood A, Khenata R et al 2016 Bull. Mater. Sci. 391419

[30] Wyckoff R W G 1986 Crystal structures, 2nd edition (USA: Krieger)

[31] Wang S Q and Ye H Q 2002 J. Phys.: Condens. Matter 14 9579

[32] Hellwege K H, Medelung O and Bornstein L 1982 (New Series Group III) Numerical data and functional relationship in science and technology (New York: Springer)

[33] Kalvoda S, Paulus B, Flude P and Stoll H 1997 Phys. Rev. B 55 4027

[34] Vancamp P E, Vandoren V E and Deverse J T 1991 Phys. Rev. B 449056

[35] Min I B, Massida S and Freeman A J 1988 Phys. Rev. B 38 1970

[36] Agarwal B K, Yadav P S, Kumar S and Agarwal S 1995 Phys. Rev. B $\mathbf{5 2} 4896$

[37] Staroverov V N and Scuseria G E 2004 Phys. Rev. B 69075102

[38] Fillipi B, Singh D J and Umrigar C J 1994 Phys. Rev. B 50 14947
[39] Paulus B, Fulde P and Stoll H 1996 Phys. Rev. B 542556

[40] Rushton P P, Clark S J and Tozer D J 2001 Phys. Rev. B 63 115206

[41] Vubcevich M 1972 J. Phys. Status Solidi B 54219

[42] Huai S and Zunger A 1999 Phys. Rev. B 605404

[43] Causa M, Dovesi R and Roetti C 1991 Phys. Rev. B 4311937

[44] Senger R T, Tongay S, Durgun E and Ciraci B 2005 Phys. Rev. B 72075419

[45] Rohlfing M, Kruger P and Pollmann J 1993 Phys. Rev. B 48 17791

[46] Alouani M and Christensen N E 1988 Phys. Rev. B 371167

[47] Arnaud B and Alouani M 2001 Phys. Rev. B 63085208

[48] Ahmad I, Amin B, Maqbool M, Muhammad S, Murtaza G, Ali S et al 2012 Chin. Phys. Lett. 29097102

[49] Khan I, Ahmad I, Amin B, Murtaza G and Ali Z 2011 Physica B 4062509

[50] Murtaza G, Ahmad I, Amin B, Afaq A, Maqbool M, Maqssod J et al 2011 Opt. Mater. 33553

[51] Xu B, Li X, Sun J and Yi L 2006 Eur. Phys. J. B 66483

[52] Babu K E, Murali N, Swamy D T and Veeraiah V 2014 Bull. Mater. Sci. 37287

[53] Rabina O, Lin Y M and Dresselhaus M S 2001 Appl. Phys. Lett. 7981

[54] Rashid B, Hilal M, Khan S H and Khan A 2016 Mater. Sci. Semicond. Process. 4183 